\title{
Sortilin mediates vascular calcification via its recruitment into extracellular vesicles
}

\author{
Claudia Goettsch, ${ }^{1}$ Joshua D. Hutcheson, ${ }^{1}$ Masanori Aikawa, ${ }^{1,2}$ Hiroshi Iwata, ${ }^{1}$ Tan Pham, ${ }^{1}$ Anders Nykjaer, ${ }^{3}$ Mads Kjolby, ${ }^{3}$ \\ Maximillian Rogers, ${ }^{1}$ Thomas Michel, ${ }^{4}$ Manabu Shibasaki, ${ }^{1}$ Sumihiko Hagita, ${ }^{1}$ Rafael Kramann, ${ }^{5,6}$ Daniel J. Rader, ${ }^{7}$ Peter Libby, ${ }^{2}$ \\ Sasha A. Singh, ${ }^{1}$ and Elena Aikawa, ${ }^{1,2}$ \\ 'Center for Interdisciplinary Cardiovascular Sciences and 'Center for Excellence in Vascular Biology, Cardiovascular Division, Brigham and Women's Hospital, Harvard Medical School, Boston, Massachusetts, \\ USA. ${ }^{3}$ The Lundbeck Foundation Research Center MIND and The Danish Research Institute of Translational Neuroscience, Nordic European Molecular Biology Laboratory Partnership for Molecular Medicine, \\ and Danish Diabetes Academy, Department of Biomedicine, Aarhus University, Aarhus, Denmark. ${ }^{4}$ Cardiovascular Medicine and ${ }^{5}$ Renal Division, Brigham and Women's Hospital, Harvard Medical School, \\ Boston, Massachusetts, USA. ${ }^{6}$ Division of Nephrology, RWTH Aachen University, Aachen, Germany. ${ }^{7}$ Institute for Translational Medicine and Human Genetics, Perelman School of Medicine at the University \\ of Pennsylvania, Philadelphia, Pennsylvania, USA.
}

\begin{abstract}
Vascular calcification is a common feature of major cardiovascular diseases. Extracellular vesicles participate in the formation of microcalcifications that are implicated in atherosclerotic plaque rupture; however, the mechanisms that regulate formation of calcifying extracellular vesicles remain obscure. Here, we have demonstrated that sortilin is a key regulator of smooth muscle cell (SMC) calcification via its recruitment to extracellular vesicles. Sortilin localized to calcifying vessels in human and mouse atheromata and participated in formation of microcalcifications in SMC culture. Sortilin regulated the loading of the calcification protein tissue nonspecific alkaline phosphatase (TNAP) into extracellular vesicles, thereby conferring its calcification potential. Furthermore, SMC calcification required Rab11-dependent trafficking and FAM20C/casein kinase 2-dependent C-terminal phosphorylation of sortilin. In a murine model, Sort1-deficiency reduced arterial calcification but did not affect bone mineralization. Additionally, transfer of sortilin-deficient BM cells to irradiated atherosclerotic mice did not affect vascular calcification, indicating a primary role of SMC-derived sortilin. Together, the results of this study identify sortilin phosphorylation as a potential therapeutic target for ectopic calcification/microcalcification and may clarify the mechanism that underlies the genetic association between the SORT1 gene locus and coronary artery calcification.
\end{abstract}

\section{Introduction}

Cardiovascular calcification correlates with cardiovascular risk factors. Arterial calcification, particularly when presented as microcalcifications in the fibrous cap, predicts cardiovascular events $(1,2)$. Clinical imaging studies also identified spotty calcification as a marker of plaques prone to cause acute coronary syndrome (3). Moreover, computational modeling indicated that microcalcification increases mechanical stress at the surface of thin-capped atheroma implicated in plaque rupture $(4,5)$. Despite its considerable clinical impact, no medical therapies exist to prevent or treat cardiovascular calcification.

While conventional views recognized calcification as a degenerative disorder during aging, recent evidence has established that calcification remains an active process - smooth muscle cells (SMCs) undergo phenotypic modulation, acquire the expression of osteogenic proteins, and deposit a mineralized bone-like matrix (6). SMC-induced calcification involves the release of calcifying extracellular vesicles (EVs), a subpopulation traditionally known as matrix vesicles (7). We recently demonstrated that EVs contribute to the development of microcalcification (8); however, the

Conflict of interest: M. Shibasaki and S. Hagita are employees of KOWA Pharmaceutical, Tokyo, Japan.

Submitted: January 7, 2015; Accepted: January 21, 2016.

Reference information: J Clin Invest. 2016;126(4):1323-1336. doi:10.1172/JCI80851. precise mechanisms of the formation of EVs with high calcification potential and their role in SMC arterial calcification remain unclear. A better understanding of the molecular mechanisms of cardiovascular calcification, particularly the derivation of calcifying EVs and microcalcification, may lead to the development of novel therapeutic strategies.

GWAS found that the 1p13 locus harboring the SORT1 gene encoding the protein sortilin associated with plasma low-density lipoprotein (LDL) cholesterol levels (9), myocardial infarction (10), aortic aneurysm (11), and coronary artery calcification (12). The multiligand sorting receptor sortilin has functional characteristics of the vacuolar protein sorting 10-protein domain family (13). In addition to its protein-sorting functions in the brain (14), sortilin regulates lipid metabolism and inflammation. The global deletion of sortilin reduces secretion of very low-density lipoprotein $(\mathrm{VLDL})$ from the liver $(15,16)$ and reduces atherosclerosis in mice $(17,18)$.

This study demonstrates a previously unknown function of sortilin - promoting calcification via its trafficking function of tissue nonspecific alkaline phosphatase (TNAP) to EVs that leads to high mineralization competence in the extracellular milieu. Our in vivo findings led to a plausible notion that SMC-derived sortilin may play a direct role in ectopic calcification, independent of potential remote effects as a consequence of its lipid metabolism functions in the liver. We demonstrate that the phos- 
phorylation status of the intracellular domain of sortilin regulates the protein trafficking in the cell, which reveals the pathological impact of one specific C-terminal phosphoserine. Our results point to sortilin as a therapeutic target for hindering calcification/microcalcification, a common cardiovascular disorder with no specific preventive therapy.

\section{Results}

Sortilin levels increase in calcified human atheroma and in calcified arteries from patients and experimental animals with chronic renal disease (CRD). IHC of human atheromata obtained from carotid endarterectomy demonstrated extensive expression of sortilin in von Kossa-stained calcified regions (Figure 1A). Sortilin expression largely localized to $\alpha \mathrm{SMA}$-positive cells but not to regions of CD68-positive cells (Figure 1, A and B, and Supplemental Figure 1A; supplemental material available online with this article; doi:10.1172/JCI80851DS1). More specifically, sortilin expression is associated with microcalcifications, as demonstrated with a near-infrared fluorescent (NIRF) calcium tracer $\left(\mathrm{R}^{2}=0.59, P<0.0001\right)$ (Figure $\left.1 \mathrm{C}\right)$.

This study also examined decalcified femoral arteries from patients with CRD and demonstrated that they express sortilin in the aSMA-positive tunica media (Supplemental Figure 1B). We previously showed that CRD accelerates both intimal and medial calcification in apolipoprotein E-deficient $\left(\right.$ Apoe $\left.^{-/}\right)$mice (19). These data therefore triggered the assessment of the vascular expression of sortilin in $A p o e^{-/-}$mice fed a high-fat diet for 20 weeks and in Apoe $e^{-/-}$mice with CRD induced by 5/6 nephrectomy. Sortilin expression highly associated with calcification, as demonstrated by TNAP activity and von Kossa staining, as well as with immunoreactive osteopontin (Figure 1D and Supplemental Figure 2A). Results showed negligible sortilin expression in the vessel wall of WT mice compared with Apoe ${ }^{-/}$mice (Supplemental Figure 2, B and C). In the aortic media of $\mathrm{Apoe}^{-/-}$mice with CRD, the sortilin-immunopositive area showed a 6.6-fold greater increase compared with Apoe $^{-/-}$(Figure 1, D and E) mice. CRD increased the intimal sortilin-positive area by 3.6-fold compared with $A_{p o e^{-/}}$mice with intact kidneys $(P=0.029)$ (Figure $1, \mathrm{D}$ and E). Correspondingly, the whole aortic lysate of CRD mice had

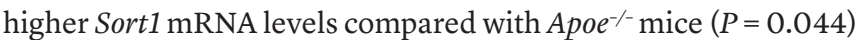
(Supplemental Figure 2D).

The isolation of sortilin-expressing SMCs from $A p o e^{-/-}$mice using laser-capture microdissection (Supplemental Figure 2, E and F) and the performance of mRNA expression profiling of osteogenic markers helped determine whether sortilin expression associates with the calcifying SMC phenotype. Sortilin-expressing SMCs exhibited lower mRNA levels of Acta2 $(P=0.026)$ and higher levels of $A l p l(P=0.014)$ (Figure 1F) and Bglap $(P=0.04)$ (Supplemental Figure $2 \mathrm{G}$ ) compared with SMCs that do not express sortilin. These data support our findings in calcified human atheroma, in which SMCs exhibit key osteogenic makers including runtrelated transcription factor 2 (RUNX2), osteocalcin, and osteopontin in association with high sortilin expression (Supplemental Figure 3). In summary, sortilin is associated with vascular calcification in human and mouse atheromata.

Sortilin deficiency reduces vascular but not skeletal calcification in vivo. The study of Sort1 $1^{+/+}$and Sort1/-- mice helped identify the in vivo effect of sortilin on vascular calcification (Supplemental Figure 4). Aortas from Sort1 $1^{-/-}$mice and Sort1 ${ }^{+/+}$littermate controls were explanted and cultured in osteogenic media. The ex vivo approach permits calcification of C57BL/6 mice arteries, which otherwise harbor resistance to calcification in vivo. At 14 days of ex vivo culture, Sort $1^{+/+}$mice exhibited TNAP activity in the tunica media, whereas $\operatorname{Sort1}^{-/-}$mice did not show any changes (Figure 2A).

Next, we assessed the effects of genetic deletion of sortilin in LDL receptor-deficient mice ( $\left(\mathrm{dll} \mathrm{r}^{-/}\right)$consuming a high-fat, highcholesterol diet - conditions conducive to vascular calcification (19). Sort $1^{-/} \mathrm{Ldlr}^{-/-}$mice exhibited reduced vascular calcification when compared with Sort1 ${ }^{+/+} \mathrm{Ldlr}^{-/}$control littermates, as demonstrated by fluorescence reflectance imaging (FRI) of a NIRF calcium tracer in intact arteries (Figure 2B and Supplemental Figure $5 \mathrm{~A})$. Quantification revealed an $80 \%$ reduction in total aortic calcification area in $\mathrm{Sort1}^{-/-} \mathrm{Ldlr/-}$ mice compared with Sort1 ${ }^{+/+} \mathrm{Ldlr}^{-/}$ mice $(P=0.003)$ (Figure 2C). Moreover, Sort1 ${ }^{-/-} \mathrm{Ldlr}^{-/-}$mice showed reduced extracted aortic calcium mineral $(P=0.048)$ (Figure $2 \mathrm{D})$. Histological analysis revealed less TNAP activity (Figure 2, E and F), calcium deposition (von Kossa staining, Supplemental Figure 5B), and arterial microcalcification (Supplemental Figure 5C)

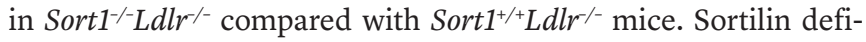
ciency did not alter serum cholesterol levels when mice consumed a high-cholesterol diet for 15 weeks (Supplemental Figure 5D). Sort1 ${ }^{-/-} \mathrm{Ldll}^{-/-}$mice demonstrated reduced serum cholesterol levels when they consumed a Western-type diet, as previously reported (15). Sort1 $1^{-1-} \mathrm{Ldlr}^{-/}$mice consuming the Western high-fat, $0.25 \%$ cholesterol diet also displayed reduced vascular calcification (diminished von Kossa and alizarin red S staining), microcalcification, and vascular calcium (Supplemental Figure 6, A-D).

Although CD68 did not coexpress with sortilin in calcified human atheroma (Figure 1B), this study further explored a potential role of immune cell-derived sortilin in vascular calcification. We transplanted BM from Sort ${ }^{+/+}$or Sort ${ }^{-/-}$mice to lethally irradiated $\mathrm{Ldlr}^{-/}$mice and subjected them to a high-fat, high-cholesterol diet for 24 weeks. Total cholesterol and root lesion size did not change (Supplemental Figure 7). Deficiency of sortilin in cells in the hematopietic lineage $\left(\mathrm{Sort}^{-/-} \rightarrow \mathrm{Ldlr}^{-/-}\right.$) did not alter vascular calcification as demonstrated by FRI imaging (Figure 2, G and $\mathrm{H}$ ), quantitative vascular calcium (Figure 2I), TNAP activity, or microcalcification staining (Supplemental Figure 7, D and E). Moreover, sortilin protein remained in the calcific lesions in mice that received Sort ${ }^{-/-}$BM cells (Supplemental Figure 7F), indicating that sortilin derived from immune cells (e.g., T cells, macrophages) may not contribute substantially to sortilin-mediated vascular calcification.

Two types of mouse experiments evaluated the effect of sortilin on bone morphology: evaluation of 10-week-old Sort $1^{-/-}$mice and $\operatorname{Sort}^{+/+}$littermate controls that consumed a chow diet, and Sort1 $^{-/-} \mathrm{Ldlr}^{-/}$and Sort1 ${ }^{+/+} \mathrm{Ldlr}^{-/}$littermate controls that consumed a high-fat, high-cholesterol diet, as described above. In both genotypes, microCT analysis of the femur revealed similar bone mass at the cancellous region of the distal femur and the cortical shaft (Figure 3, A and B, and Supplemental Tables 1 and 2). Sortilin deficiency altered neither cortical and trabecular bone volume and thickness, nor trabecular number (Figure 3, C-G). 

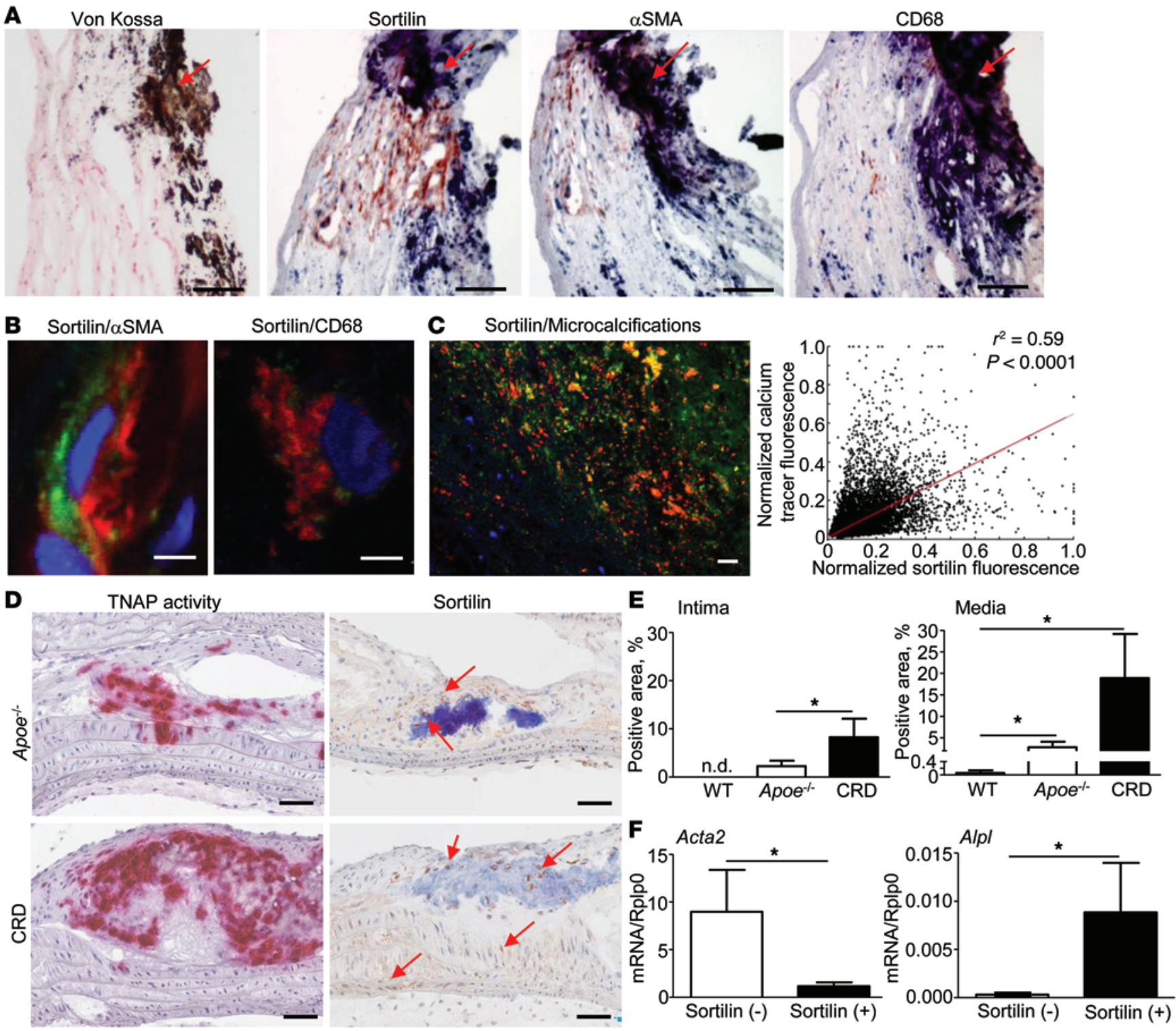

Figure 1. Sortilin localizes to calcified tissue from humans and mice with CRD. (A) von Kossa staining indicates calcified areas (red arrow). Calcified regions of human atherosclerotic carotid arteries contain sortilin. SMCs are stained with $\alpha$ SMA and macrophages with CD68. Scale bars $100 \mu \mathrm{m}$. (B) Immunofluorescence double staining of sortilin (green)/ $\alpha$ SMA (red) or CD68 (red). One representative sample of 20 patients. Scale bars: $5 \mu \mathrm{m}$. (C) Immunofluorescent visualization of sortilin and microcalcifications using near infrared fluorescence (NIRF) calcium tracer in human atherosclerotic carotid arteries. Scale bars: $20 \mu \mathrm{m}$. Graph: data points represent correlation between sortilin and tracer signal from 10 patients. Each image was separated into 250 smaller images for quantification using a total of 2,500 measurements. (D) Immunohistochemical staining of sortilin (brown reaction product, red arrows) in aorta of $A p o e^{-/-}$ mice without and with CRD. TNAP activity detects calcification-prone tissue. One of 4 animals per group is shown. Scale bars: $100 \mu \mathrm{m}$. (E) Quantification of sortilin-positive cells in atherosclerotic lesions and media. $n=3$, WT, CRD; $n=4, A p o e^{-1}$. n.d., not detected for WT. ${ }^{*} P<0.05$, ANOVA. Error bars indicate \pm SD. (F) Acta2 and Alpl mRNA expression from sortilin negative (-) and positive (+) SMCs (laser capture microdissection of $A p o e^{-/-}$aorta). $n=7 .{ }^{*} P<0.05$, Wilcoxon matched-pairs signed rank test. Error bars indicate \pm SEM.

Furthermore, static and dynamic bone histomorphometry demonstrated no effect of genetic Sort deletion on bone osteoblast and osteoclast number and mineral apposition rate (Supplemental Figure 8, A-C). Next, BM cells from Sort1 ${ }^{+/+}$or Sort1 ${ }^{-/}$mice were allowed for ex vivo osteoblast or osteoclast differentiation experiments. Sortilin deficiency did not alter osteoclast (Supplemental Figure 8, D-G) or osteoblast (Supplemental Figure $8, \mathrm{H}-\mathrm{K}$ ) function and marker gene expression profiles. Sortilin silencing also did not affect human osteoblastogenesis
(Supplemental Figure 8, L-O). Taken together, these data indicate that sortilin plays a causal role in vascular calcification but does not alter bone mineralization.

Sortilin directly affects mineralization of human vascular SMCs. Human coronary arterial SMCs (hSMCs) cultured in osteogenic medium, which can induce hSMC calcification (20), showed a time-dependent increase in sortilin mRNA and protein abundance (Figure 4A and Supplemental Figure 9, A and B). Calcifying hSMCs exhibited sortilin levels comparable to those of human hepatocytes 
A
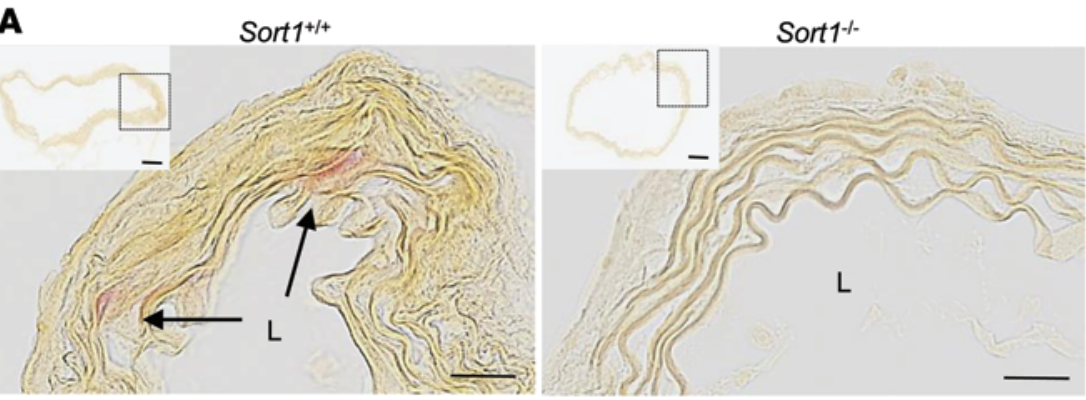

B Sort ${ }^{+1+} L d l r^{1-}$

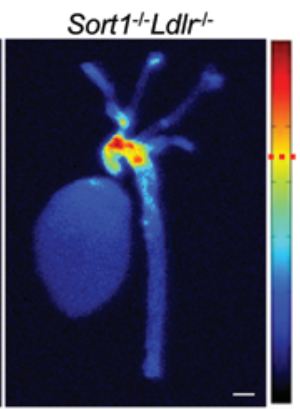

C

Osteosense FRI

D

Aortic calcium
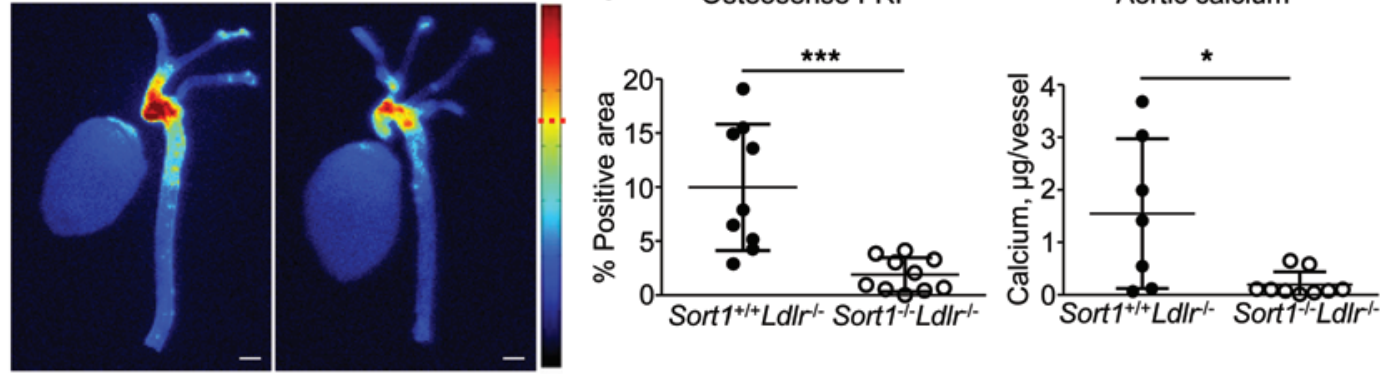

E

Sort $1^{+/+}$Ldlr'-

Sort ${ }^{1-}$ Ld dIr ${ }^{\text {- }}$

$\mathbf{F}$

TNAP activity
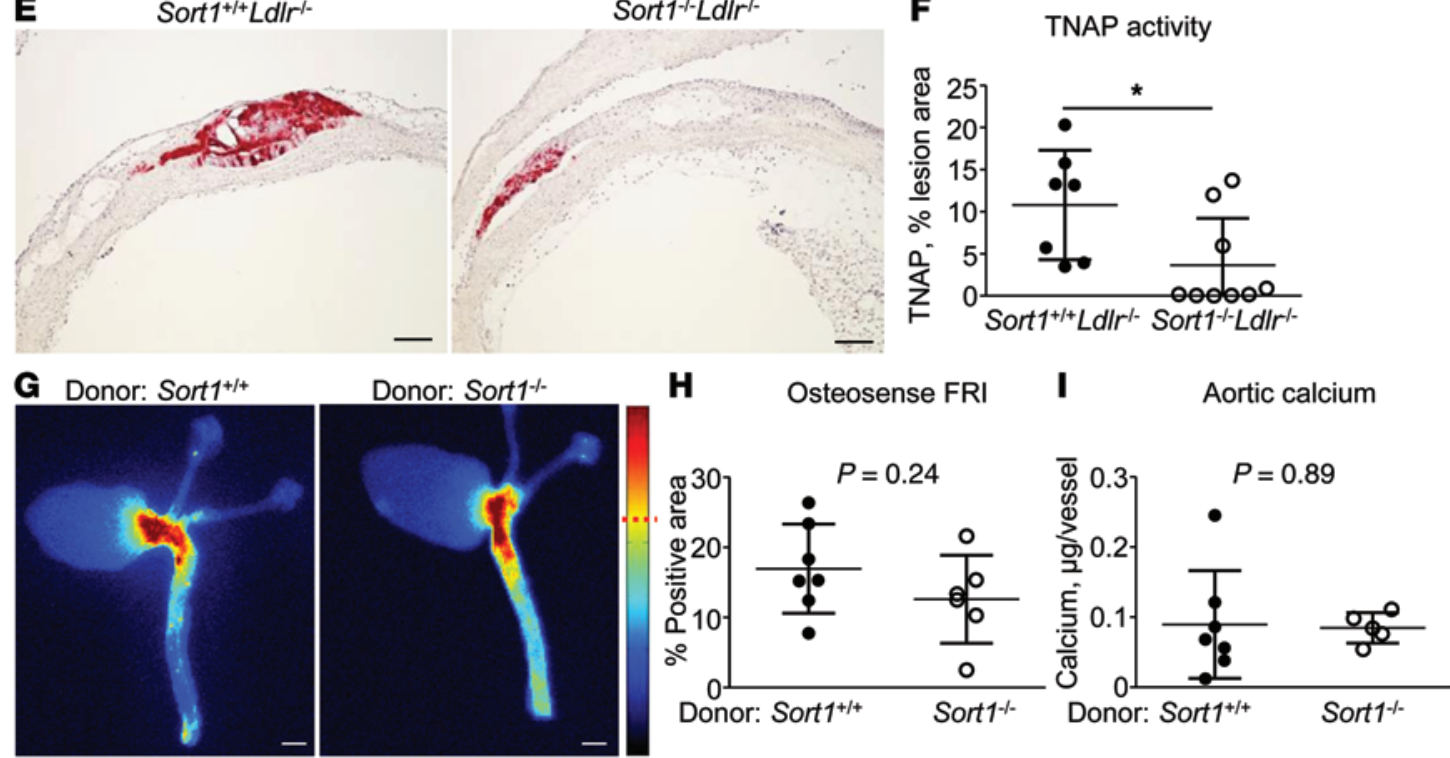

Figure 2. Sortilin is necessary for vascular calcification in vivo. (A) TNAP activity (arrows) after ex vivo aortic ring culture of Sort $7^{+/+}$littermates and Sort $7^{-/-}$

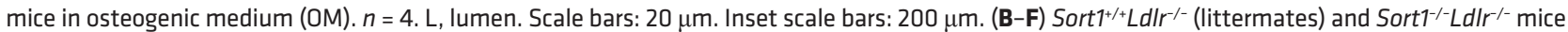
(10-week-old, sex-mixed) consumed a high-fat, high-cholesterol (1.25\% cholesterol) diet for 15 weeks. (B) Ex vivo FRI analysis. Representative images of the fluorescence intensity in the aorta. Scale bars: $2 \mathrm{~mm}$. (C) Quantification of FRI signal-positive area (dashed red line indicates signal cut-off for quantification)

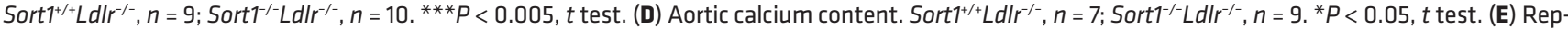
resentative images of TNAP activity in the aortic arch (lesser curvature). Scale bars: $20 \mu \mathrm{m}$. (F) Quantitative assessment of TNAP by percent-positive lesion

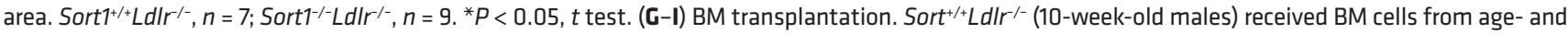
sex-matched Sort ${ }^{+/}$and Sort ${ }^{-/}$mice and consumed a high-fat, high-cholesterol $(1.25 \%$ cholesterol) diet for 24 weeks. (G) Ex vivo FRI analysis. Representative images of the fluorescence intensity in the aorta. Scale bar: $2 \mathrm{~mm}$. (H) Quantification of FRI signal-positive area. Donor: Sort $1^{+/+}, n=7 ;$ Sort1 ${ }^{-/-}, n=6$. (I) Aortic calcium content. Donor: Sort $7^{+/+}, n=7$; Sort $1^{-/}, n=5$. Each dot depicts one mouse. Error bars indicate \pm SD.

expressing high levels of sortilin (ref. 9 and Supplemental Figure 9C). Immunofluorescence localized sortilin in areas of microcalcifications, as detected by NIRF calcium tracer (Figure 4B).

Loss-of-function and gain-of-function experiments sought to establish whether sortilin plays a direct role in SMC calcification (Supplemental Figure 9, D-F). Silencing sortilin reduced TNAP activity $(-30 \%, P=0.009)$ in calcifying hSMCs (Figure
4C). Increasing endogenous sortilin using adenoviral overexpression promoted TNAP activity 2 -fold $(P=0.015)$ (Supplemental Figure 9G). Sortilin silencing decreased matrix calcification (Supplemental Figure $9 \mathrm{H}$ ), as demonstrated by a $33 \%$ reduction of incorporated calcium $(P=0.004$ ) (Figure $4 \mathrm{D})$, and sortilin overexpression enhanced calcification by $43 \%$ $(P=0.002)$ (Supplemental Figure 9I). Modulation of sortilin did 
A
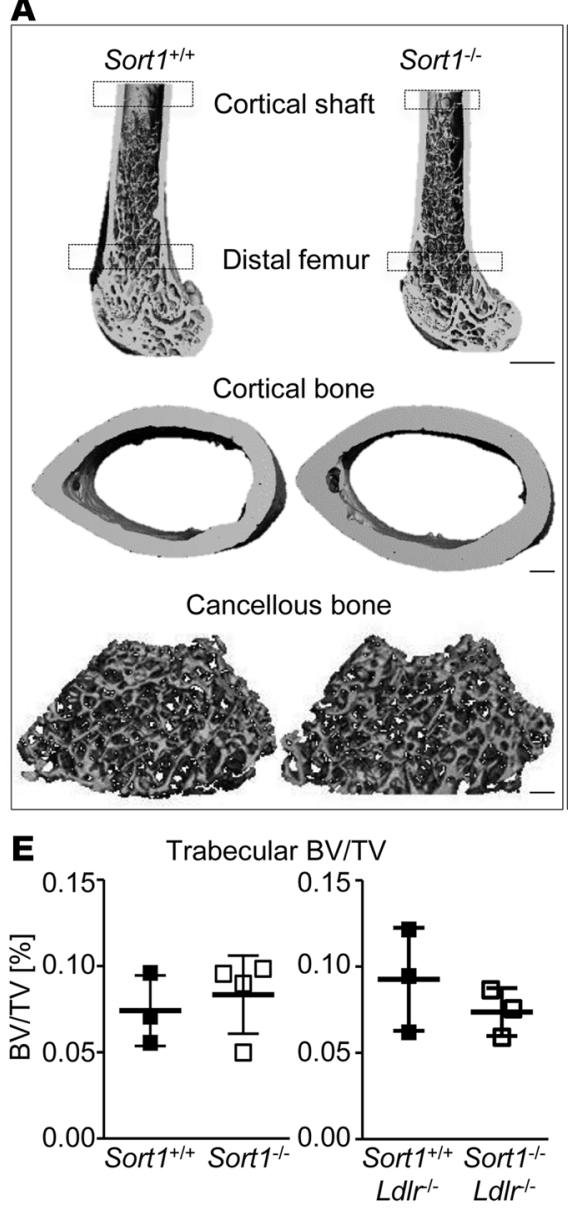

B
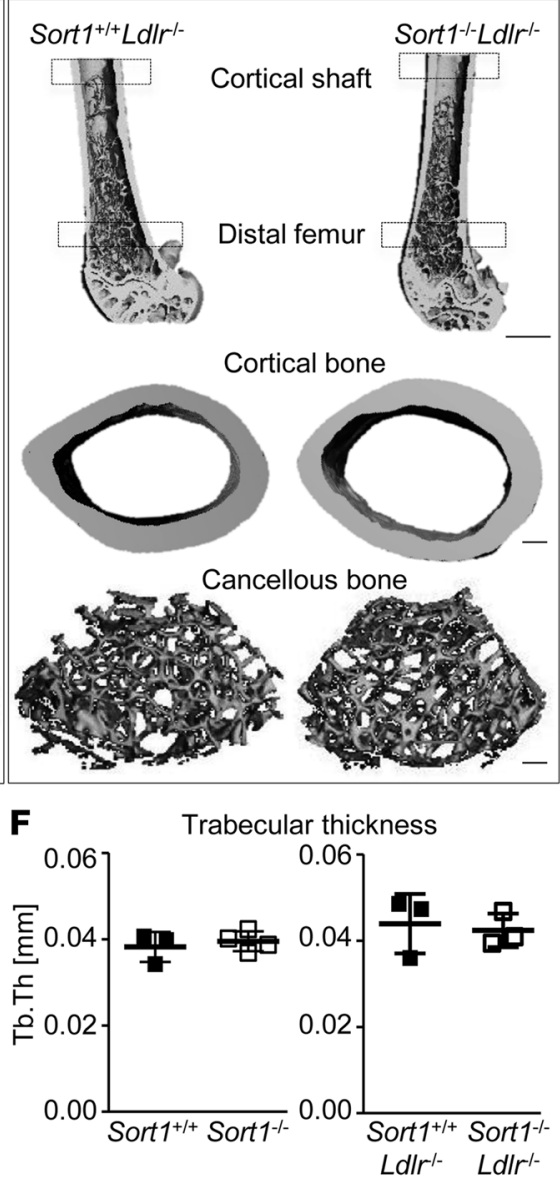

C
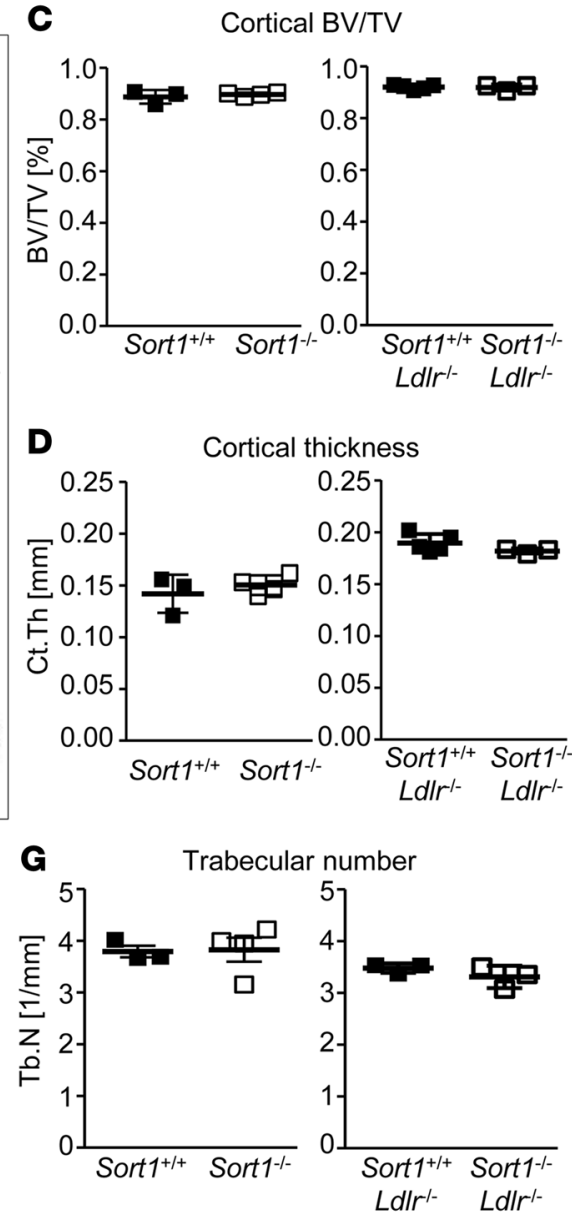

Figure 3. Sortilin deficiency does not affect bone structure in mice. (A and $\mathbf{B}$ ) Cortical and trabecular bone was assessed by microCT. Representative bone cross sections from 10 -week-old female Sort $1^{+/+}$littermates and Sort1 ${ }^{-/-}$mice that consumed a chow diet (A) and 25 -week-old female Sort $1^{+/+}$Ldlr $^{-/-}$littermates and Sort1 ${ }^{-/-}$Ldl/ ${ }^{-/-}$on a high-fat, high-cholesterol diet for 15 weeks (B). Scale bars: 1 mm for longitudinal bone sections; 200 $\mu \mathrm{m}$ for regional cross sections. (C and E) Bone volume/tissue volume (BV/TV). (D) Cortical thickness. (F) Trabecular thickness. (G) Trabecular number. $+/+, n=3 ;-/-, n=3-4$. Each dot depicts 1 mouse. Error bars indicate \pm SD.

not affect total cholesterol levels in calcifying hSMCs (Supplemental Figure 10, A and B).

Prior studies implicated apoptosis and osteogenic reprogramming in arterial calcification (7). Sortilin silencing and overexpression did not affect cellular viability (Supplemental Figure 10C), nor did it change the expression of the key osteogenic transcription factor RUNX2 (Supplemental Figure 10, $\mathrm{D}$ and E). Furthermore, a PCR array on mRNA extracted from calcifying hSMCs with sortilin silenced by siRNA helped analyze the expression of 84 transcripts related to osteogenesis. Modulation of sortilin did not change mRNA levels that encode SMAD and SOX9, critical factors for osteochondrogenic differentiation (data not shown). These data indicate that sortilin may promote calcification by acting downstream of osteochondrogenic reprogramming.

Sortilin associates with calcifying EVs. Recent studies indicated that SMC-induced calcification proceeds through the release of calcifying EVs (21). Transmission electron microscopy showed accumulation of EVs associated with microcalcification in atheromata of mice with CRD (Figure 4E). Electron microscopy-based immunogold staining detected sortilin in calcified EVs of human atheromata (Figure 4F). Western blot analyzed EVs isolated from calcifying hSMCs and showed enhanced sortilin and TNAP signals when compared with controls (Figure 4G). Parallel reaction monitoring, a targeted mass spectrometry method (22), verified the presence of sortilin peptides in calcifying hSMC-derived EVs (Supplemental Figure 11, A and B).

An investigation into the potential role of sortilin in $\mathrm{EV}$ release and mineralization competence then followed. Enforced expression of sortilin in hSMCs increased loading of sortilin into EVs, which increased further in EVs derived from calcifying hSMCs (Supplemental Figure 11C). Nanoparticle-tracking analysis, in combination with sortilin loss-of-function and gain-of-function experiments, indicated that the number of EVs and their size distributions do not depend on sortilin loading (Supplemental Figure 12, A-D). Silencing of cellular sortilin-reduced TNAP activity within EVs $(-45 \%, P<0.001)$ (Figure $4 \mathrm{H})$, whereas cellular sortilin overexpression promoted EV TNAP activity 2 -fold $(P=0.045)$ (Figure $4 \mathrm{I}$ ), suggesting that the loading of sortilin into the EVs promotes their mineralization potential. 

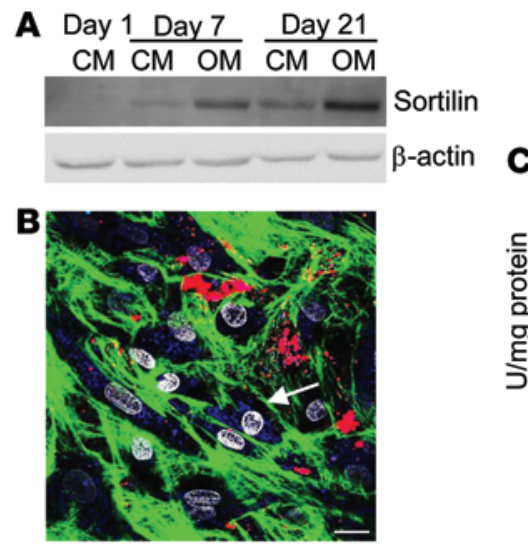

$\mathbf{E}$

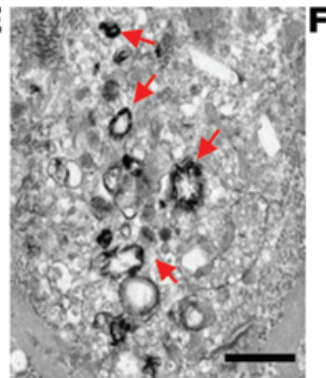

$\mathbf{H}$

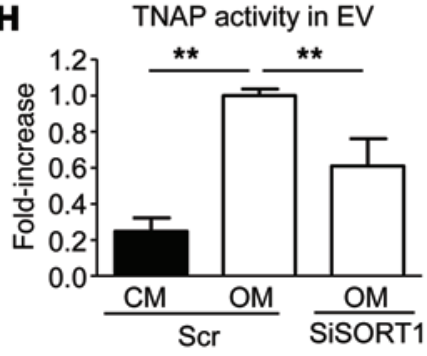

D
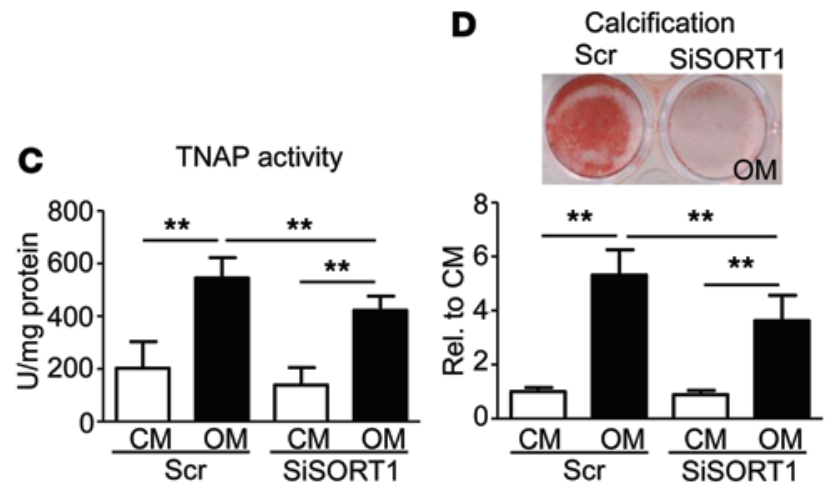

Sortilin immunogold

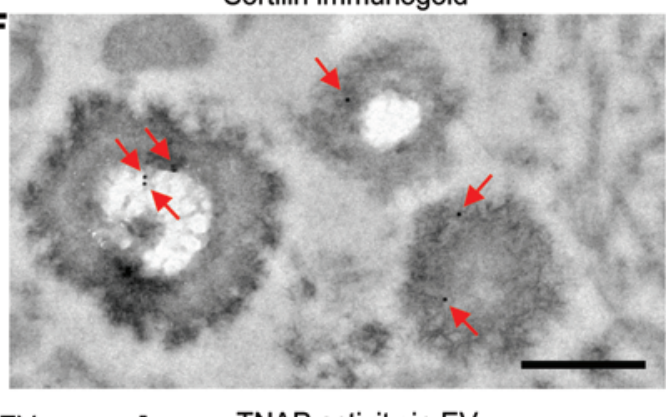

G EV

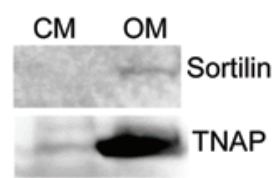

Figure 4. Sortilin promotes alkaline phosphatase activity and mineralization in calcifying hSMCs and EVs. hSMCs were cultured up to 21 days in control medium (CM) or osteogenic medium (OM). (A) Relative sortilin protein abundance. Representative Western blot $(n=3)$. (B) Immunofluorescence microscopy of sortilin (blue, arrow) in areas of microcalcifications (red) at day 21. Collagen, green; nuclei, gray. Scale bar: $20 \mu \mathrm{m}$. (C and D) Sortilin was silenced by siRNA (siSORT1 or scramble control, Scr). $n=4 .{ }^{* *} P<0.01$, ANOVA. (C) TNAP activity at day 14. (D) Calcification at day 21. Top: representative images of calcification detected by alizarin red S-stained mineralized matrix. Bottom: quantification of eluted calcium. (E) EVs of different sizes and appearance (red arrows) including small loci of microcalcifications. Calcified plaque of CRD mouse. Scale bar: $1 \mu \mathrm{m}$. (F) Transmission electron microscopy-based immunogold staining of sortilin (red arrows) in EVs from aorta of CRD mouse. One of 3 animals is shown. Scale bar: $200 \mathrm{~nm}$. (C) Endogenous sortilin and TNAP levels in EVs isolated from hSMC supernatant. $n=3$. (H and I) TNAP activity in EVs isolated from hSMCs cultured for 14 days in CM or OM. Sortilin was silenced by siRNA (siSORT1 or scramble control, Scr) or overexpressed by adenovirus (AdSORT1 or LacZ control). $n=3,{ }^{* *} P<0.01$, ANOVA (H) and $n=4,{ }^{*} P<0.05, t$ test (I). Error bars indicate \pm SD. Each $n$ indicates an independent hSMC donor.

Caveolin-1 and TNAP are key interactors of sortilin in calcifying $h S M C s$ and EVs. The presence of sortilin in EVs prompted the hypothesis that interacting proteins congregate with sortilin in EVs to control calcification. Mass spectrometric examination of the changes in the sortilin interactome in calcifying hSMCs revealed an increase of coimmunoprecipitated TNAP and caveolin-1, compared with control hSMCs (Figure 5, A and B; Supplemental Figure 13, A-E; and Supplemental Table 3).

The enrichment of caveolin-1 in the calcifying condition suggested a role for specific caveolin-enriched membrane/lipid rafts (CEMs) in the release of the calcification-prone EVs. Electron microscopy of aorta sections of Apoe $e^{-/}$mice with CRD demonstrated an association of caveolae and calcification (Figure 5C). Moreover, caveolin-1 and sortilin colocalized on the plasma mem- brane of calcifying hSMCs (Supplemental Figure 13F). Electron microscopy-based immunogold staining showed sortilin on the plasma membrane of SMCs within calcified human atherosclerotic plaques (Supplemental Figure 13G). To investigate whether SMC calcification affects the cellular redistribution of sortilin to CEMs, which play an essential role in the regulated exocytosis pathway $(23,24)$, the present study used discontinuous sucrose density gradients to resolve CEM from other cellular constituents unassociated with CEM (nCEM). Caveolin-1, a positive control, showed CEM enrichment (Figure 5D). In calcifying hSMCs, sortilin redistributed into CEM fractions, suggesting the translocation of sortilin within specialized membrane domains during SMC calcification (Figure 5D and Supplemental Figure 14, A and B). Calcifying hSMCs and their released EVs showed increased cave- 
A
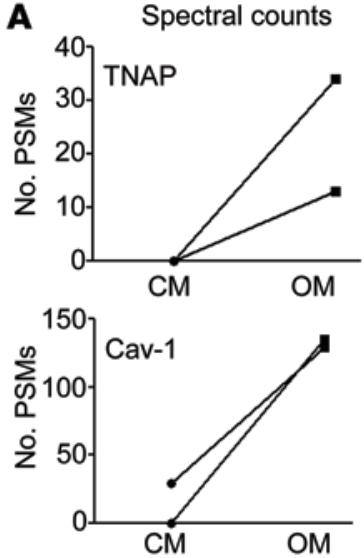

B

Sortilin

TNAP

Cav-1

Sortilin

TNAP

Cav-1

\section{F Calcification}
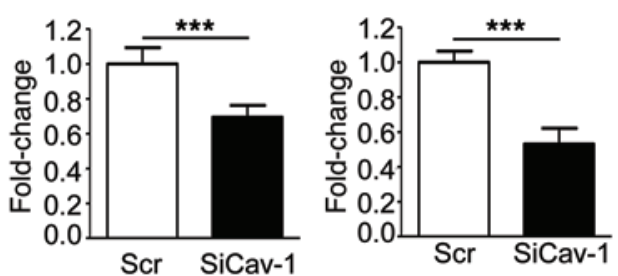

G
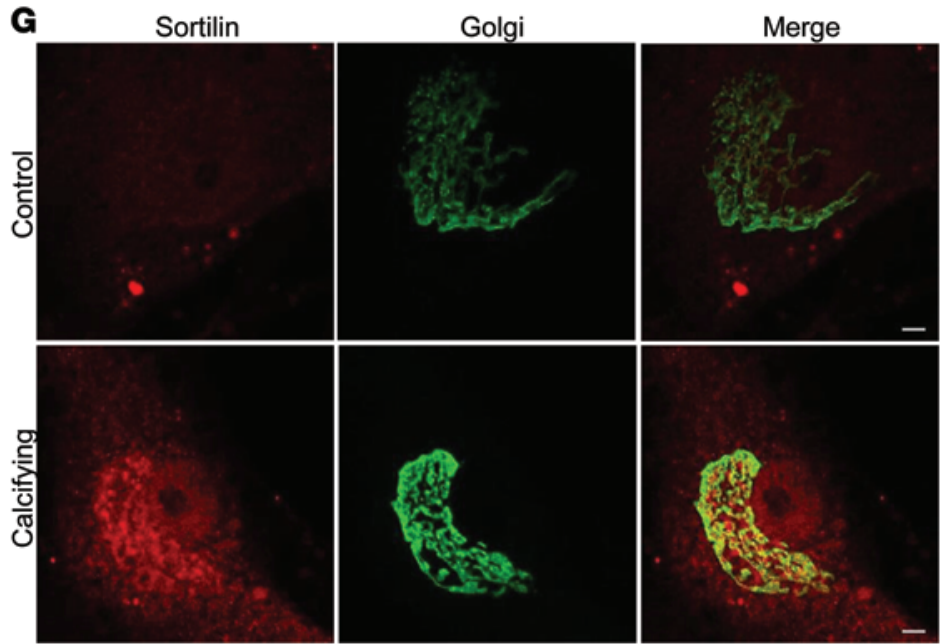

H

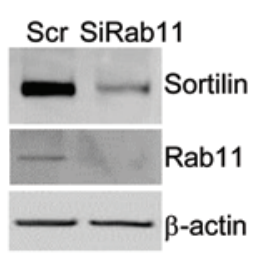

I
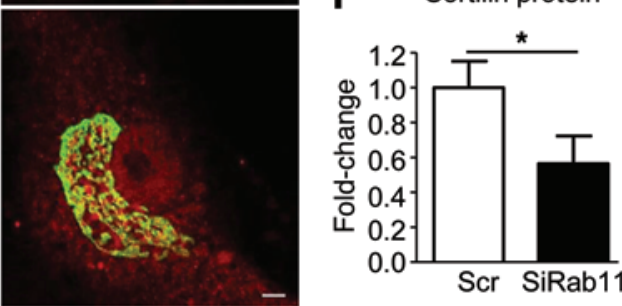

$$
\text { J }
$$

TNAP activity

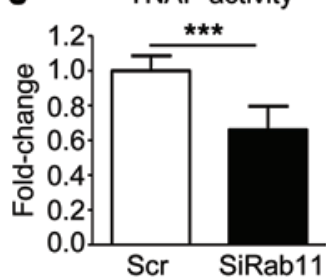

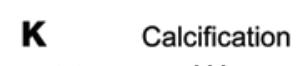

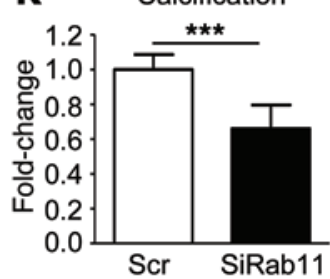

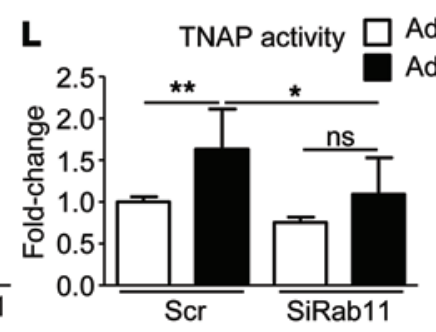

Cav-1

Sortilin

TNAP
Figure 5. TNAP interacts with sortilin in calcifying SMCs. Rab11 controls sortilin trafficking. (A) Number of peptide spectrum matches (PSM) from caveolin-1 (Cav-1) and TNAP identified in sortilin immoprecipitation experiments. $n=3$. (B) Western blot of sortilin, Cav-1, and TNAP before (input) and after IP of sortilin. One of 3 hSMC donors is shown. (C) Transmission electron microscopy of aorta sections of CRD Apoe - $^{-/}$ mice. HA, hydroxyapatite. Scale bar: $2 \mu \mathrm{m}$. Inset shows a higher magnification depicting caveolae (red arrows). Scale bar: $200 \mathrm{~nm}$. (D) hSMCs were cultured for 21 days in control medium (CM) or osteogenic medium (OM). CEM was resolved from other cellular constituents (nCEM). Representative Western blot, $n=3$. (E and $\mathbf{F}$ ) hSMCs were cultured for 14 days (E, TNAP activity) or 21 days ( $F$, calcium eluted from the matrix) in OM. Cav-1 was silenced by siRNA (SiCav-1 or scramble control, Scr). $n=4 .{ }^{* *} P<0.005, t$ test. (G) Immunofluorescence staining of sortilin (red) and the TCN (GALTN2, green) in control and calcifying hSMCs. Scale bar: $10 \mu \mathrm{m}$. (H-L) Rab11a/b were silenced by siRNA (SiRab11). (H) Representative Western blot of sortilin and Rab11. $\beta$-Actin served as loading control. (I) Quantification of H. $n=3$. ${ }^{*} P<0.005, t$ test. (J) TNAP activity at day 14. (K) Calcification quantified by measuring calcium eluted from the matrix, $n=3$. ${ }^{* * *} P<0.005, t$ test. (L) Sortilin was overexpressed by adenovirus (AdSORT1). LacZ served as control (AdLacZ). $n=3$. ${ }^{*} P<0.05,{ }^{*} P<$ 0.01 , ANOVA. Error bars indicate \pm SD. Each $n$ indicates an independent hSMC donor. olin-1 levels (Supplemental Figure 14, C and D). Silencing of caveolin-1 reduced sortilin protein levels in whole cell lysates from calcifying hSMCs (Supplemental Figure 14, C and E), as well as in the CEM fractions (Supplemental Figure 14E). Silencing of caveolin-1 in hSMCs reduced TNAP activity $(-31 \%, P<0.001)$ (Figure $5 \mathrm{E})$ and calcification $(-47 \%, P<0.001)$ (Figure $5 \mathrm{~F}$ ). SMCs isolated from caveolin-1-deficient mice exhibited decreased calcification and no change in TNAP activity (Supplemental Figure 14, F and $G)$. These data suggest that osteogenic cues redistribute sortilin and TNAP to specialized membrane domains, leading to their loading into EVs. The content of these EVs may therefore affect their calcification potential.

Rab11 regulates sortilin-dependent calcification. Intracellular protein trafficking influences the loading of proteins into EVs. Trans-Golgi network-to-plasma (TGN-to-plasma) membrane transport requires Rab11, which regulates the transport of recycling endosomes (25). TNAP activates in the Golgi apparatus (26). This consideration engendered the hypothesis that Rab11 regulates sortilin-dependent calcification through trafficking sortilin and activated TNAP from the Golgi to the plasma membrane for 
loading into EVs. Sortilin redistributes to the TGN of calcifying hSMCs, as shown by immunocytochemical analysis (Figure 5G). Silencing of Rab11a/b reduced sortilin protein by $50 \%$ (Figure 5 , $\mathrm{H}$ and I, and Supplemental Figure $14 \mathrm{H}$ ) and also reduced TNAP activity (Figure 5J) and calcification (Figure 5K). Further, silencing Rab11 abolished the induction of TNAP activity by sortilin overexpression (Figure 5L), suggesting a Rab11-dependent pathway as a trafficking route involved in sortilin-induced calcification.

Phosphorylation of sortilin C-terminus accelerates calcification. Posttranslational modification affects the trafficking of proteins (27). Thus, a mass spectrometry-based strategy monitored posttranslational modification of sortilin (28-30). Our immunoprecipitationstudiesrevealed phosphorylation atserine 825(SGYHDD [pS] DEDLLE, pSer825), which is located within the C-terminal intracellular domain tail of sortilin (Figure 6A). A global phosphoproteomic study previously described phosphorylation at Ser825 (31), but prior work has not implicated this modification in vascular calcification. The establishment of a targeted mass spectrometry quantification strategy, enabled by an in vitro-synthesized (29) phosphorylated form of sortilin (Supplemental Figure 15A), determined a correlation between pSer825 and calcification. Relative quantification of the MS1 peaks of unmodified versus phosphorylated pSer825 revealed basal phosphorylation at Ser825 at day 7 for hSMCs in control and calcifying conditions. By day 14, however, the relative abundance of pSer825 increased 3-fold and the unmodified peptide concomitantly decreased 3-fold in calcifying cells, compared with their relative abundances in control cells (Figure 6B and Supplemental Figure 15B).

Further experiments assessed pSer825 in mouse and human calcified arteries. Parallel reaction monitoring of unmodified versus phosphorylated pSer825 revealed significantly higher modified C-terminal peptide in calcifying arteries compared with noncalcifying vessels harvested from $A_{p o e^{-/-}}$mice $(P=0.005)$ (Figure $6 \mathrm{C}$ and Supplemental Figure $15 \mathrm{C}$ ). Calcified human carotid endarterectomy samples showed $23.8 \% \pm 10.7 \%$ of pSer825, whereas nonatherosclerotic carotid artery specimens obtained at autopsy had $7.9 \% \pm 5.5 \%$ of pSer825 $(P=0.039)$ (Figure 6D).

In silico searches for phosphorylation of the S-X-E motif suggested family with sequence similarity 20, member C (Fam20C), as a kinase involved in the Ser825 phosphorylation of sortilin. hSMCs express Fam20C (Supplemental Figure 16A). Kinase assays using either the C-terminal peptide standard (Figure 6E) or membrane protein isolated from sortilin-overexpressing HEK293 cells (Figure 6F) revealed that Fam20C phosphorylates sortilin at Ser825. A catalytically inactive Fam20C form (D478A) exerted no modification (Figure 6, E and F). Overexpression of Fam20C in calcifying hSMCs increased pSer825 $(P=0.029)$ (Figure 6G) and promoted TNAP activity $(P=0.008)$ that was diminished by sortilin silencing (Figure $6 \mathrm{H}$ ). Fam20C overexpression did not alter TNAP protein (Supplemental Figure 16B). Fam20C silencing did not affect TNAP activity (Supplemental Figure 16C), indicating the involvement of additional kinases. In silico analysis revealed casein kinase 1 (CK1) and CK2 as candidate kinases that could phosphorylate Ser825 (Supplemental Table 4). Previous studies have reported the phosphorylation of Ser825 by CK2 (32). Indeed, in our experiments CK2, but not CK1, increased pS825 in a cellfree kinase assay (Figure 6I).
Further experiments aimed to determine the relative contribution of Fam20C and CK2 in phosphorylating sortilin on Ser825 in calcifying hSMCs. We performed a kinase assay with the sortilin C-terminal peptide standard (SGYHDDSDEDLLE) using cell lysates as sources for protein kinases. Compared with the control lysate, the calcifying hSMC lysate demonstrated an increased propensity to phosphorylate the sortilin peptide $(P=0.005)$ (Supplemental Figure 16D). Fam20C overexpression further increased this propensity $(P=0.019)$, while additional CK2 inhibition by tetrabromobenzotriazole (TBB) did not alter it (Supplemental Figure 16E). Silencing of Fam20C in calcifying hSMCs, in combination with CK2 inhibition, demonstrated a reduced propensity to phosphorylate Ser825 compared with scramble vehicle control (Figure 6J) and thus reduced TNAP activity (Figure 6K). These data suggest that Fam20C and CK2 are both sufficient to promote sortilin-dependent calcification.

The introduction of mutations at Ser825 to prevent phosphorylation (alanine; S825A) or to mimic a constitutive active phosphorylation (aspartic acid; S825D) helped to investigate the functional relevance of the C-terminal serine site (Figure 7A). Transduction of mutated constructs did not alter cellular sortilin protein concentrations compared with nonmutated constructs (Figure 7B). Enforced expression of S825A prevented the sortilin-dependent increase in cellular TNAP activity (Figure 7C) and calcification (Figure 7D), whereas S825D further enhanced TNAP activity (Figure 7C) compared with the overexpression of the nonmutated sortilin. S825A also mitigated the sortilin-dependent increase of TNAP activity in hSMC-derived EVs (Figure 7E), while the number of released EVs did not change (Supplemental Figure 16F). Furthermore, mutation of Ser825 modified the intracellular localization of sortilin. While sortilin with constitutively active Ser825 phosphorylation (S825D) localized to the TGN, sortilin with the null-phosphorylation (S825A) colocalized with Lamp1, suggesting trafficking to the endolysosomal system for degradation (Figure $7 F$ ). These data indicate that sortilin phosphorylation controls its trafficking and subsequently facilitates the transport of TNAP into EVs, thereby enhancing their mineralization competence.

\section{Discussion}

Sortilin recently gained attention due to human genetic and lipid metabolism studies $(9,12,15,16)$. GWAS associated a variation in the sortilin locus with cardiovascular calcification (12). It remains unknown, however, how the single nucleotide polymorphisms associate with sortilin levels in the calcified vasculature. This new study identified sortilin as a key regulator of vascular calcification, a condition currently lacking therapy.

Recent reports revealed a contribution of SMC-derived EVs to calcification (7). The present study unraveled a sortilin-mediated pathway that promotes the calcification potential of EVs derived from SMCs in vitro and validated the relevance of our mechanistic findings in vivo. Our findings demonstrated high sortilin expression in human calcified carotid and femoral arteries and in calcified atherosclerotic lesions of mice. Sortilin-deficient mice developed less arterial calcification while their bone structure and bone cell function remained unchanged, indicating the sortilin-associated mineralization pathology is specific to the vasculature. A previous study revealed that sortilin regulates the inflammatory status of 

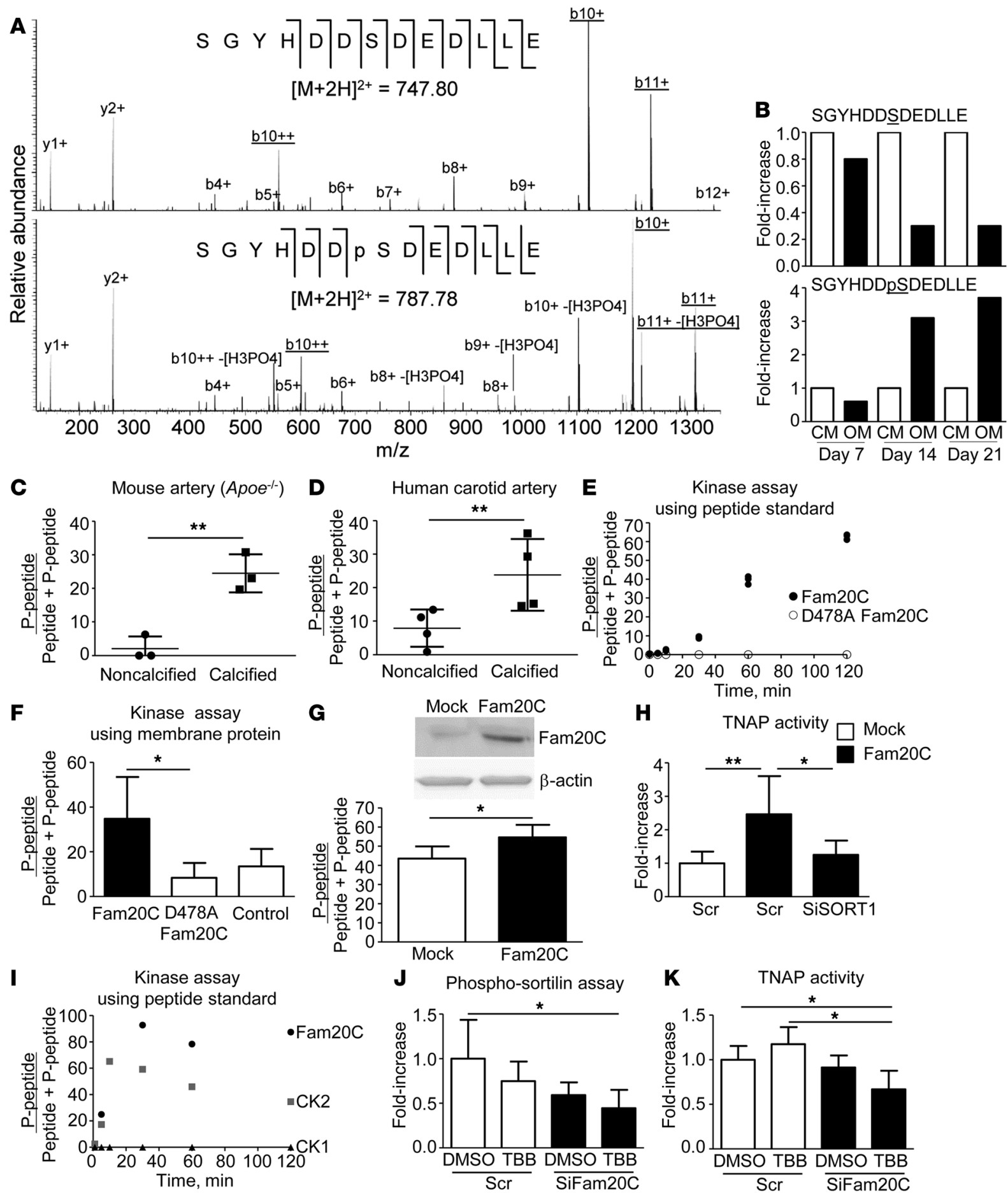

J Phospho-sortilin assay

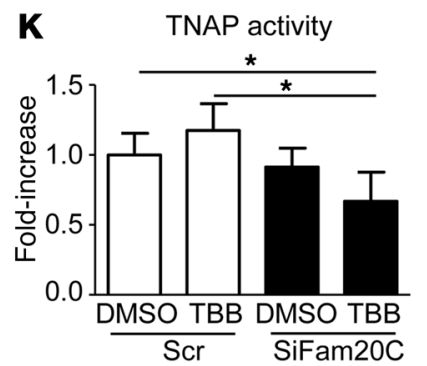

Figure 6. Ser825 of sortilin C-terminus is phosphorylated in calcifying hSMCs and in vivo. (A) MS/MS spectrum for SCYHDDSDEDLLE $\left([M+2 H]^{2+}=747.80\right)$, and phospho-peptide, pS825 $\left([\mathrm{M}+2 \mathrm{H}]^{2+}=787.78\right)$. (B-G) The underlined fragment ions were used to determine the percent of phospho-peptide in samples using parallel reaction monitoring (PRM). (B) Relative ratio of phosphorylated (bottom) and unphosphorylated (top) form. Fold-increase over control medium (CM) is plotted. (C) pS825 in calcified and noncalcified artery from Apoe $e^{-/-}$mice. $n=3$. Each $n$ correspond to pooled artery from 3 mice. ${ }^{*} P<0.05, t$ test. (D) pS825 in human calcified carotid arteries from endarterectomy and noncalcified arteries from autopsy. $n=4$. ${ }^{*} P<0.05, t$ test. (E) Kinase assay using peptide standard (SCYHDDSDEDLLE). Time-dependent phosphorylation by Fam20C or catalytically inactive form D478A. (F) Kinase assay using membrane protein isolated from sortilin-overexpressing HEK293 cells. Membrane protein without enzyme incubation served as control. $n=3 .{ }^{*} P<0.05, t$ test. (G) pS825 in calcifying hSMCs overexpressing Fam20C. Empty vector served as control (Mock). Top: Fam20C Western blot. $\beta$-Actin served as loading control. $n=3$. ${ }^{*} P<0.05$, paired $t$ test. (H) TNAP activity after Fam20C overexpression and sortilin silencing (SiSORT1). $n=3 .{ }^{* *} P<0.01,{ }^{*} P<0.05$, ANOVA. (I) Time-dependent phosphorylation by Fam20C, CK1, and CK2. (J) Kinase assay using peptide standard. Cell lysates from calcifying hSMCs were used as a kinase source. Fam20C was silenced. TBB $(10 \mu \mathrm{M})$ was used to inhibit CK2. DMSO served as solvent control. (I and J) Quantification was done using the $M S 1$ ion signals. $n=4 .{ }^{*} P<0.05$, ANOVA. (K) TNAP activity. ${ }^{*} P<0.05$, ANOVA. Error bars indicate $\pm S D$. Each $n$ indicates an independent hSMC donor for $\mathbf{G}, \mathbf{H}, \mathbf{J}$, and $\mathbf{K}$. OM, osteogenic medium. 
A agtggttatcatgatgactcagatgaggaCCTCtTGgAatAG $\begin{array}{llllllllllllll}\text { S } & G & Y & H & D & D & S_{825} & D & E & D & L & L & E & \underline{s t o p}\end{array}$

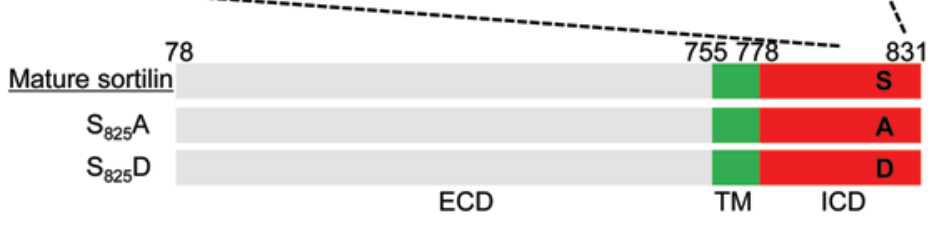

B

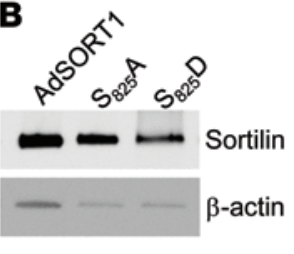

C

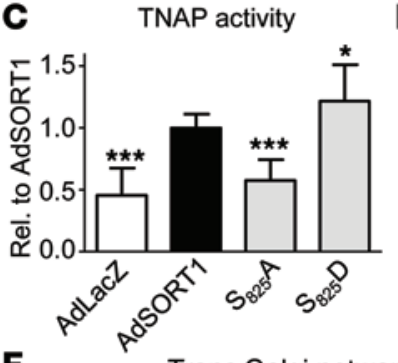

$\mathbf{F}$
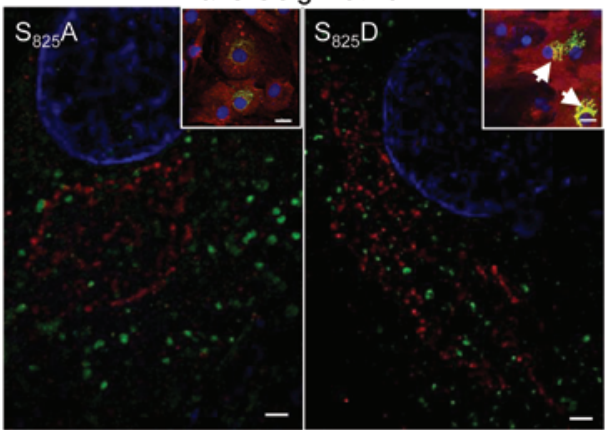

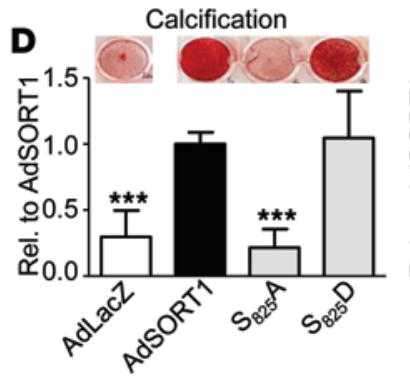

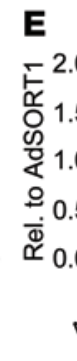

TNAP activity in EV

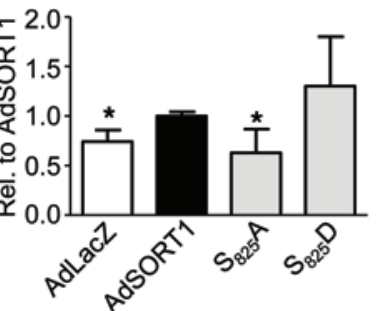

Lysosome

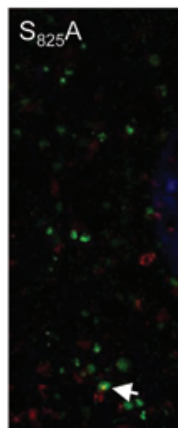

Figure 7. Phosphorylation of the sortilin C-terminus accelerates calcification. (A) Strategy for mutation of the C-terminal peptide. The numbers indicate amino acid positions. ECD, extracellular domain; TM, transmembrane domain; ICD, intracellular domain. (B-F) hSMCs were transduced with adenoviral vectors to overexpress sortilin (AdSORT1) or the different mutated forms (phosphorylation-null S825A; phosphorylation-mimetic S825D). Adenovirus LacZ served as control (AdLacZ). (B) Representative sortilin Western blot of calcifying hSMCs with transduced mutated constructs. $\beta$-Actin served as loading control. (C) TNAP activity. $n=4$. (D) Calcification. Top: representative alizarin red $\mathrm{S}$ staining. Bottom: Quantification of eluated alizarin red $S, n=4$. (E) TNAP activity in EVs. TNAP activity was normalized to EV protein content. $n=3$. ${ }^{*} P<0.05$, ${ }^{* *} P<0.005$ vs. AdSORT 1 by $t$ test for all experiments. Error bars indicate \pm SD. (F) Immunofluorescence using super-resolution microscopy (insets: confocal microscopy) shows the expression of sortilin (green) and TCN (GALTN2, red) or lysosome (LAMP1, red) in hSMCs overexpressing sortilin 5825A or 5825D. Arrows indicate coexpression. Scale bars: $2 \mu \mathrm{m}$; inset scale bars: $20 \mu \mathrm{m}$. Each $n$ indicates an independent hSMC donor. Representative of 3 independent experiments. (G) Schematic representation of the mechanism of sortilin-induced calcification via C-terminus phosphorilation (yellow), Rab1-dependent trafficking, and TNAP loading into EV. 
macrophages, thus promoting atherosclerotic lesion formation (17). The present study's results obtained from BM transplantation experiments suggest a role of sortilin on vascular calcification that is independent of immune cell-derived sortilin and therefore possibly mediated by SMC-derived sortilin. While current data show that sortilin affects hepatic lipoprotein secretion $(9,15,16)$, emerging evidence suggests that sortilin contributes to cardiovascular disease development independently of circulating cholesterol $(11,17)$, thus supporting our data that show no changes in cholesterol levels associated with sortilin deficiency in vascular calcification in mice.

In mediating formation of calcifying EVs, sortilin traffics through the TGN, undergoes phosphorylation, and thereby facilitates the transport of TNAP via Rab11-enriched endosomes to specialized caveolin-1-expressing membrane domains. The sortilin/TNAP complex then enters EVs to augment their calcification potential (Figure 7G). Thus, the data support the hypothesis that SMC-derived EVs actively contribute to vascular calcification and provide the distribution pathway by which TNAP is exported to the extracellular membrane.

The evidence suggests that TNAP activity is sufficient to drive arterial calcification (33). TNAP activity converts pyrophosphate into phosphate (34) and is necessary for matrix vesicle-dependent bone mineralization (35), while its role in EV-dependent vascular calcification remains unclear (36). TNAP-negative vesicles derived from exosomal origins may participate in hyperphosphatemicinduced SMC calcification associated with CRD $(21,37)$, whereas TNAP-positive SMC-derived vesicles may exert the osteogenic actions observed in atherosclerosis (38). The regulation of TNAP activity in calcifying SMC-derived EVs, however, remains poorly understood. The present study shows that sortilin and its posttranslational modification following osteogenic phenotypic modulation regulates the calcification competence and TNAP activity of SMC-derived EVs. Silencing of sortilin decreased TNAP activity in SMCs and SMC-derived EVs. Sortilin C-terminus phosphorylation controls loading of activated TNAP into EVs. Of note, a previous in vitro study revealed sortilin as a promotor of osteoblastogenesis without affecting TNAP activity (39). Our observations elucidated a calcification-dependent association of sortilin and TNAP in the lipid raft/caveolin-enriched membrane and thereby supports the known function of sortilin as a sorting coreceptor that fine-tunes signaling by ligand-dependent trafficking of specific receptors (40). Further studies will elucidate the sequence of events related to sortilin-TNAP interactions within caveolinenriched membranes, cellular TNAP activation, TNAP loading into calcifying EVs, and potential feedback mechanisms that alter transcriptional regulation of calcification-related proteins.

We described the Rab11-dependent recycling pathway as a trafficking route involved in sortilin-dependent calcification. Rab11 orchestrates the intersection between endocytic- and exocytic-trafficking pathways (41). Silencing Rab11 decreased sortilin protein and TNAP activity, indicating the participation of Rab11 transport of these proteins to the plasma membrane, rendering them susceptible to lysosomal degradation. Rab11 may therefore regulate the trafficking of sortilin and associated TNAP activation via recycling endosomes, which regulates calcification.

Proteomic analysis confirmed phosphorylation of S825 (31, 32), positioned within the C-terminal tail of sortilin (cytoplasmic domain). A variety of mass spectrometry tools helped demonstrate an increase in propensity for pS825 in SMC calcification in vitro and in vivo. Previous studies have demonstrated that CK2 targets S825 with functional significance $(32,42)$. The present study confirmed these findings in vascular calcification and extended the functional kinase list for Ser825 to include Fam20C. Fam20C, a Golgi complex-associated CK and key regulator of osteoblast mineralization (43), phosphorylates secretory pathway type I and II proteins within S-X-E motifs (44). Enforced expression of Fam20C in SMCs indeed increased pS825 and promoted calcification.

The essential role of specialized lipid raft domains in the regulation of the exocytosis pathway $(23,24)$ engendered the hypothesis that the intracellular domain controls the loading of sortilin into EVs. Gain- and loss-of-function phospho-mutants help demonstrate that the phosphorylation status of Ser825 directs the cellular localization of sortilin. Previous work demonstrated that phosphorylation of the cation-independent mannose 6-phosphate receptor on the cytoplasmic tail, which has high sequence similarity to sortilin, directly associates with its exit from the TGN (45). Previous studies indicate that, while the extracellular domain of sortilin associates with direct receptor interactions, the intracellular domain regulates its biological effects (46). The present study, which shows that sortilin-dependent calcification requires posttranslational modification of the C-terminus, supports this notion. The S825 phosphomimetic activated TNAP activity through TGN localization, while the phosphor-null variant localized to lysosomes. These data agree with previous findings that TNAP activation occurs in the early secretory pathway and subsequently moves and localizes to the plasma membrane (26) and that the sortilin intracellular domain controls the fate of interacting partners from the lysosomes to lipid rafts (47). The p 825 site enhances the binding of sortilin-to-Golgi-associated, $\gamma$-adaptin ear-containing, ARF-binding protein (GGA) - cytosolic adaptors mediating the sorting of transmembrane proteins (48).

Recent reports demonstrated the participation of sortilin in exosome release (49), which raises the possibility that sortilin mediates the type of EVs rather than their cargo. Loss-offunction and gain-of-function studies, however, did not reveal a sortilin-dependent change in number and size of EVs from calcifying SMCs. Whether the release of sortilin-enriched calcifying EVs occurs via exosomal mechanisms through multivesicular bodies (37) or via budding of vesicles directly from the plasma membrane and the characteristics of sortilin/TNAP-containing EVs remains unknown. Thus, sortilin operates via a mechanism involving direct modulation of the calcification potential of EVs by facilitating the intracellular trafficking and loading of TNAP into the EVs.

In conclusion, this study revealed a previously unknown function of sortilin in vascular calcification and provided a potential mechanistic insight into the GWAS, which associated the SORT1 gene with coronary artery disease (50), particularly coronary artery calcification (12). Studies have indicated that sortilin-associated SNPs correlate with tissue expression in an asymmetric manner $(9,11)$. For example, although one particular SNP decreased sortilin levels in the liver (9), sortilin levels in vascular tissue did not correlate with this SNP (11). The final evidence for the causal tripartite association among genetic variations of the sortilin locus, changes in vascular sortilin expression, and ectopic calcification 
remains obscure. Additional studies using databases of genetic studies involving the collection of vascular tissue can elucidate this direct genetic role of sortilin in coronary calcification.

Conventional view has attributed cardiovascular calcification to passive tissue degeneration during aging. Current concepts view calcification as the result of highly regulated pathological pathways, potentially subject to intervention. Although this common disorder contributes to several clinical conditions (e.g., plaque rupture, aortic stenosis), medical therapies remain unavailable. Due to the aging of the population, vascular calcification will increase as a global health burden, driving our investigations. The mechanistic insight provided in the present study has identified sortilin as a therapeutic target for the prevention or treatment of calcification without affecting bone mineralization.

\section{Methods}

Further information can be found in Supplemental Methods.

Human primary coronary artery SMC culture and osteogenic transition. hSMCs (PromoCell) were grown in SMC growth medium 2 (SMC-GM2, PromoCell) supplemented with epidermal growth factor $(0.5 \mathrm{ng} / \mathrm{ml})$, insulin $(5 \mu \mathrm{g} / \mathrm{ml})$, basic fibroblast growth factor-B $(2 \mathrm{ng} / \mathrm{ml})$, and FBS (5\%). Cells were used between passages 3 and 8 . Cells from at least 3 independent donors were used.

hSMCs were cultured for up to 21 days in the presence of either control medium (DMEM, 10\% FBS, 1\% penicillin/streptomycin) or osteogenic medium (consisting of control medium supplemented with $10 \mathrm{nM}$ dexamethasone, $10 \mathrm{mM} \beta$-glycerol phosphate, and 100 $\mathrm{mM}$ L-ascorbate phosphate). Medium was changed 3 times per week.

Animal procedures. Sort ${ }^{-/}$mice were generated by targeted deletion of $191 \mathrm{bp}$ of exon 14 encoding for part of the $\beta$-propeller domain (genOway). Mice were bred in our animal facility $\left(\mathrm{Ldll}^{/} /\right.$mice were obtained from The Jackson Laboratory).

In all experiments, littermate control mice were used. Experiments were carried out on 10-week-old male and female mice consuming a high-fat, high-cholesterol diet (1.25\% cholesterol, D12108C, Research Diets Inc.) for 15 weeks. Sort $1^{-/-}$and $\mathrm{Ldlr}^{-/}$mice consuming a Western-type diet have been reported previously (15).

Ex vivo aortic explant experiment. Sort ${ }^{+/+}$and Sort ${ }^{-/}$mice were sacrificed, and aortas were prepared as follows: fat and adventitia were removed from the aorta and cut into rings; to induce calcification, aortic rings were incubated in osteogenic medium as described above for hSMCs for 14 days; and the aortic rings were snap-frozen and analyzed for TNAP activity.

Visualization of microcalcification. Microcalcification was detected using a bisphosphonate-conjugated imaging agent that binds to hydroxyapatite (OsteoSense 680, PerkinElmer), elaborating fluorescence evident though the near-infrared window (ex/em 650/680 nm) as previously described (8). Briefly, frozen sections were treated with OsteoSense 680 (1:100) overnight before imaging or further immunofluoresence staining.

Isolation and quantification of EVs $(30-300 \mathrm{~nm})$. Culture medium (0.1\% FBS) conditioned by hSMCs for 24 hours underwent centrifugation at $1,000 \mathrm{~g}$ for 5 minutes to remove cell debris. The EV fraction was harvested from the media by ultracentrifugation at $100,000 \mathrm{~g}$ for 40 minutes at $4^{\circ} \mathrm{C}$ (Optima Max Ultracentrifuge, Beckman Coulter). Nanoparticle Tracking Analysis using a Nanosight LM10 measured EVs between $30-300 \mathrm{~nm}$. For protein isolation and TNAP activity,
EVs were washed with PBS followed by another ultracentrifugation at $100,000 \mathrm{~g}$ for 40 minutes at $4^{\circ} \mathrm{C}$. EVs were either lysed in protein lysis buffer or TNAP lysis buffer.

Statistics. Data are given as mean $\pm \mathrm{SD} ; n$ indicates the number of independent experiments. Statistical analyses were performed using the GraphPad Prism program (Prism Software Inc., Version 5). For comparison between two groups, a paired or unpaired two-tailed Student's $t$ test with equal or unequal variances was performed. For comparison among three or more treatment groups, one-way ANOVA followed by Bonferroni's post test was done. Exclusion criteria were set by Grubbs test. No statistical method was used to predetermine sample size. CRD animal experiments were randomized. The quantitative analyses for immunofluorescence and histology were performed blinded to allocation during outcome assessment. Correlation analyses were performed according to Pearson. P values less than 0.05 were considered statistically significant.

Study approval. Atherosclerotic carotid arteries were collected from patients undergoing endarterectomy procedures and carotid arteries from autopsies at Brigham and Women's Hospital according to IRB protocols 1999P001348 and 2013P002517/BWH. CRD femoral arteries were obtained from consented clinical autopsies of dialysis patients from RWTH Aachen University, Germany, according to the ethical vote EK 180/14.

All animal experiments were approved by and performed in compliance with Beth Israel Deaconess Medical Center's Institutional Animal Care and Use Committee (protocol 017-2010) or were approved by the Danish Animal Experiments Inspectorate (protocol J. 2006/561-1206).

\section{Author contributions}

CG designed the study, prepared samples, conducted experimental work, acquired data, carried out data interpretation, and drafted the manuscript. JDH aided with imaging experiments and image analyses, data interpretation, and manuscript development. MA was responsible for funding, participated in data interpretation, and provided critical review of the manuscript. HI performed animal experiments and aided with data interpretation. TP performed calcification experiments and data acquisition. AN and MK provided animal tissue and participated in data interpretation. MR performed cholesterol analysis and participated in data interpretation. TM directed the caveolin-1 experiments and participated in data interpretation. MS and SH aided with animal experiments. RK provided human CKD specimens and participated in data interpretation. PL provided human carotid atherosclerotic specimens and critically reviewed the manuscript. SAS participated in the design of mass spectrometry experiments and aided in data interpretation and manuscript preparation. DJR participated in data interpretation and provided critical review of the manuscript. EA contributed to the study concept, helped with the study design, participated in data interpretation, supervised the study, and aided in overall manuscript development.

\section{Acknowledgments}

The authors thank Jack E. Dixon (UCSD, La Jolla, California, USA) for providing the Fam20C protein and the control protein. We thank Iwao Yamada, Jung Choi, Andrew Mlynarchik, and Christopher Duke for technical assistance and Sara Karwacki and Chelsea Swallom for their editorial expertise. 
This study was supported by a research grant from Kowa Company Ltd. (to M. Aikawa) and by grants from the NIH (R01HL114805 and R01HL109506 to E. Aikawa; R01HL107550 to M. Aikawa; R01HL80472 to P. Libby; and R0146457 to T. Michel) and The Lundbeck Foundation (to A. Nykjaer and M. Kjolby).
Address correspondence to: Elena Aikawa or Claudia Goettsch, Cardiovascular Medicine, Brigham and Women's Hospital, 3 Blackfan Cirlce, CLS 17th floor, Boston, Massachusetts 02115, USA. Phone: 617.730.7755; E-mail: eaikawa@partners.org (E. Aikawa), cgoettsch@partners.org (C. Goettsch).
1. Martin SS, et al. Dyslipidemia, coronary artery calcium, and incident atherosclerotic cardiovascular disease: implications for statin therapy from the multi-ethnic study of atherosclerosis. Circulation. 2014;129(1):77-86.

2. Criqui $\mathrm{MH}$, et al. Calcium density of coronary artery plaque and risk of incident cardiovascular events. JAMA. 2014;11(3):271-278.

3. Joshi NV, et al. ${ }^{18} \mathrm{~F}$-fluoride positron emission tomography for identification of ruptured and high-risk coronary atherosclerotic plaques: a prospective clinical trial. Lancet. 2014;383(9918):705-713.

4. Vengrenyuk Y, et al. A hypothesis for vulnerable plaque rupture due to stress-induced debonding around cellular microcalcifications in thin fibrous caps. Proc Natl Acad Sci U S A. 2006;103(40):14678-14683.

5. Kelly-Arnold A, Maldonado N, Laudier D, Aikawa E, Cardoso L, Weinbaum S. Revised microcalcification hypothesis for fibrous cap rupture in human coronary arteries. Proc Natl Acad Sci U S A. 2013;110(26):10741-10746.

6. Bostrom KI, Rajamannan NM, Towler DA. The regulation of valvular and vascular sclerosis by osteogenic morphogens. Circ Res. 2011;109(5):564-577.

7. Shanahan CM, Crouthamel MH, Kapustin A, Giachelli CM. Arterial calcification in chronic kidney disease: key roles for calcium and phosphate. Circ Res. 2011;109(6):697-711.

8. New SE, et al. Macrophage-derived matrix vesicles: an alternative novel mechanism for microcalcification in atherosclerotic plaques. Circ Res. 2013;113(1):72-77.

9. Musunuru K, et al. From noncoding variant to phenotype via SORT1 at the 1p13 cholesterol locus. Nature. 2010;466(7307):714-719.

10. Kathiresan S, et al. Genome-wide association of early-onset myocardial infarction with single nucleotide polymorphisms and copy number variants. Nat Genet. 2009;41(3):334-341.

11. Jones GT, et al. A sequence variant associated with sortilin-1 (SORT1) on 1p13.3 is independently associated with abdominal aortic aneurysm. Hum Mol Genet. 2013;22(14):2941-2947.

12. O'Donnell CJ, et al. Genome-wide association study for coronary artery calcification with follow-up in myocardial infarction. Circulation. 2011;124(25):2855-2864.

13. Petersen CM, et al. Molecular identification of a novel candidate sorting receptor purified from human brain by receptor-associated protein affinity chromatography. JBiol Chem. 1997;272(6):3599-3605.

14. Willnow TE, Petersen CM, Nykjaer A. VPS10P-domain receptors - regulators of neuronal viability and function. Nat Rev Neurosci. 2008;9(12):899-909.

15. Kjolby M, et al. Sort1, encoded by the cardiovascu- lar risk locus 1p13.3, is a regulator of hepatic lipoprotein export. Cell Metab. 2010;12(3):213-223.

16. Strong A, et al. Hepatic sortilin regulates both apolipoprotein B secretion and LDL catabolism. J Clin Invest. 2012;122(8):2807-2816.

17. Mortensen MB, et al. Targeting sortilin in immune cells reduces proinflammatory cytokines and atherosclerosis. JClin Invest. 2014;124(12):5317-5322.

18. Patel KM, et al. Macrophage sortilin promotes LDL uptake, foam cell formation, and atherosclerosis. Circ Res. 2015;116(5):789-796.

19. Aikawa E, et al. Arterial and aortic valve calcification abolished by elastolytic cathepsin $\mathrm{S}$ deficiency in chronic renal disease. Circulation. 2009;119(13):1785-1794.

20. Goettsch C, Rauner M, Pacyna N, Hempel U, Bornstein SR, Hofbauer LC. miR-125b regulates calcification of vascular smooth muscle cells. Am J Pathol. 2011;179(4):1594-1600.

21. Kapustin AN, et al. Calcium regulates key components of vascular smooth muscle cell-derived matrix vesicles to enhance mineralization. Circ Res. 2011;109(1):e1-e12.

22. Singh SA, Miyosawa K, Aikawa M. Mass spectrometry meets the challenge of understanding the complexity of the lipoproteome: recent findings regarding proteins involved in dyslipidemia and cardiovascular disease. Expert Rev Proteomics. 2015;12(5):519-532.

23. Svensson KJ, et al. Exosome uptake depends on ERK1/2-heat shock protein 27 signalling lipid raft-mediated endocytosis negatively regulated by caveolin-1. J Biol Chem. 2013;288(24):17713-17724.

24. Valapala M, Vishwanatha JK. Lipid raft endocytosis and exosomal transport facilitate extracellular trafficking of annexin A2.J Biol Chem. 2011;286(35):30911-30925.

25. Chen W, Feng Y, Chen D, Wandinger-Ness A. Rab11 is required for trans-golgi network-toplasma membrane transport and a preferential target for GDP dissociation inhibitor. Mol Biol Cell. 1998;9(11):3241-3257.

26. Fukunaka A, et al. Tissue nonspecific alkaline phosphatase is activated via a two-step mechanism by zinc transport complexes in the early secretory pathway. J Biol Chem. 2011;286(18):16363-16373.

27. Walsh G, Jefferis R. Post-translational modifications in the context of therapeutic proteins. Nat Biotechnol. 2006;24(10):1241-1252.

28. Singh SA, Winter D, Bilimoria PM, Bonni A, Steen $\mathrm{H}$, Steen JA. FLEXIQinase, a mass spectrometry-based assay, to unveil multikinase mechanisms. Nat Methods. 2012;9(5):504-508.

29. Singh S, Springer M, Steen J, Kirschner MW, Steen H. FLEXIQuant: a novel tool for the absolute quantification of proteins, and the simultaneous identification and quantification of potentially modified peptides. J Proteome Res.
2009;8(5):2201-2210.

30. Singh S, Kirchner M, Steen JA, Steen H. A practical guide to the FLEXIQuant method. Methods Mol Biol. 2012;893:295-319.

31. Rigbolt KT, et al. System-wide temporal characterization of the proteome and phosphoproteome of human embryonic stem cell differentiation. Sci Signal. 2011;4(164):rs3.

32. Li J, Matye DJ, Li T. Insulin resistance induces posttranslational hepatic sortilin 1 degradation in mice. J Biol Chem. 2015;290(18):11526-11536.

33. Buchet R, Millan JL, Magne D. Multisystemic functions of alkaline phosphatases. Methods Mol Biol. 2013;1053:27-51.

34. Hessle L, et al. Tissue-nonspecific alkaline phosphatase and plasma cell membrane glycoprotein-1 are central antagonistic regulators of bone mineralization. Proc Natl Acad Sci U S A. 2002;99(14):9445-9449.

35. Anderson HC, et al. Impaired calcification around matrix vesicles of growth plate and bone in alkaline phosphatase-deficient mice. Am J Pathol. 2004;164(3):841-847.

36. Hutcheson JD, Goettsch C, Rogers MA, Aikawa E. Revisiting cardiovascular calcification: A multifaceted disease requiring a multidisciplinary approach. Semin Cell Dev Biol. 2015;46:68-77.

37. Kapustin AN, et al. Vascular smooth muscle cell calcification is mediated by regulated exosome secretion. Circ Res. 2015;116(8):1312-1323.

38. Hutcheson JD, et al. Enrichment of calcifying extracellular vesicles using density-based ultracentrifugation protocol. J Extracell Vesicles. 2014;3:25129.

39. Maeda S, et al. Sortilin is upregulated during osteoblastic differentiation of mesenchymal stem cells and promotes extracellular matrix mineralization. JCell Physiol. 2002;193(1):73-79.

40. Vaegter CB, et al. Sortilin associates with Trk receptors to enhance anterograde transport and neurotrophin signaling. Nat Neurosci. 2011;14(1):54-61.

41. Welz T, Wellbourne-Wood J, Kerkhoff E. Orchestration of cell surface proteins by Rab11. Trends Cell Biol. 2014;24(7):407-415.

42. Nielsen MS, et al. The sortilin cytoplasmic tail conveys Golgi-endosome transport and binds the VHS domain of the GGA2 sorting protein. EMBO J. 2001;20(9):2180-2190.

43. Tagliabracci VS, et al. Secreted kinase phosphorylates extracellular proteins that regulate biomineralization. Science. 2012;336(6085):1150-1153.

44. Tagliabracci VS, et al. A Single Kinase Generates the Majority of the Secreted Phosphoproteome. Cell. 2015;161(7):1619-1632.

45. Meresse S, Hoflack B. Phosphorylation of the cation-independent mannose 6-phosphate receptor is closely associated with its exit from the transGolgi network. J Cell Biol. 1993;120(1):67-75.

46. Skeldal S, et al. Mapping of the interaction site 
between sortilin and the $\mathrm{p} 75$ neurotrophin receptor reveals a regulatory role for the sortilin intracellular domain in p75 neurotrophin receptor shedding and apoptosis. J Biol Chem. 2012;287(52):43798-43809.

47. Yang M, et al. The intracellular domain of sortilin interacts with amyloid precursor protein and regulates its lysosomal and lipid raft trafficking. PLoS One. 2013;8(5):e63049.

48. Cramer JF, et al. GGA autoinhibition revisited. Traffic. 2010;11(2):259-273.

49. Wilson CM, et al. Sortilin mediates the release and transfer of exosomes in concert with two tyrosine kinase receptors. J Cell Sci. 2014;127(pt 18):3983-3997.

50. Samani NJ, et al. Genomewide association analysis of coronary artery disease. $N$ Engl J Med. 2007;357(5):443-453. 